\title{
Impacto de la actividad ganadera sobre el suelo en Colombia
}

\section{Livestock impact on the ground in Colombia}

\author{
María Alejandra Mora Marín ${ }^{1}$, Lucero Ríos Pescador ${ }^{2}$, Lucero Ríos Ramos ${ }^{3}$ y José Luis \\ Almario Charry ${ }^{4}$
}

\section{Resumen}

Este artículo de revisión documental denominado impacto de la actividad ganadera sobre el suelo en Colombia, proporciona la recopilación histórica del desenvolvimiento de esta actividad en el país, teniendo en cuenta, la afectación que esta actividad ha generado sobre el suelo, en donde cabe resaltar, que el sistema de pastoreo es extensivo, provocando unas modificaciones nocivas sobre las propiedades del suelo, como también, se exaltará su imagen representativa para la economía y bajo el impacto social ocasionado en las poblaciones de forma inherente al sistema productivo. Igualmente, el tipo de investigación se ciñe al descriptivo con un enfoque analítico. En este sentido, se detallará el proceso de análisis realizado a la actividad ganadera nacional y todas sus implicaciones medioambientales.

A lo que obedece la ruta metodológica, esta investigación se basa en los procesos de revisión documental, bajo la búsqueda de información relacionada al tema a tratar, luego se hace una selección de los documentos científicos, ya sean de tesis, revistas o de otros recursos investigativos, especialmente obtenidos por la vía internet; luego, se organiza la información sobre una base de datos, en donde se estructura el documento, a partir de un resumen que sintetiza los principales aspectos el artículo; la introducción sobre la importancia del tema; siguiendo con los resultados sobre los hallazgos obtenidos por expertos en la materia. Más adelante ya con los resultados constituidos se realiza una especie de análisis o comparación de los diversos planteamientos de los autores llamada Discusión, con lo que después servirá de base para emitir unas conclusiones que puntualizan los hallazgos bibliográficos, y para cerrar el documento, la bibliografía que es el listado de los estudios de donde se ha sacado la información.

Palabras clave: pastoreo extensivo; sistema productivo; efectos ambientales; deforestación; propiedades fisicoquímicas

\begin{abstract}
This article document review called livestock impact on the ground in Colombia, provides the historical record of the development of this activity in the country, taking into account the effects that this activity has generated on the ground, where it is worth noting that the grazing system is extensive, causing a harmful change on soil properties, as well, .se exalting its representative picture for the economy and the social impact caused low populations inherently productive system. Similarly, the type of research adheres to a descriptive analytical approach. In this sense, the process of analysis to domestic livestock and all its environmental implications will be detailed.
\end{abstract}

1. Ingeniera agroecóloga. Universidad de la Amazonía.Alejandramoramarin93@gmail.com

2. Ingeniera agroecóloga. Universidad de la Amazonía. Lucerorios21@ hotmail.com

3. Ingeniera agroecóloga. Universidad de la Amazonía. Rios_93@hotmail.es

4. Estudiante de Ingeniera agroecólogica. Universidad de la Amazonía. Joseluisalmariocharry@hotmail.com 
To which obeys the methodological route, this research is based on the processes of document review, in the search for information related to the topic, then a selection of scientific documents is made, whether theses, magazines or other resources research, especially obtained via the internet; then, information on a database, where the document is structured from a summary that summarizes the main aspects of the paper is organized; the introduction on the importance of the issue; Continuing with the results on the findings obtained by experts in the field. Later on with made results a kind of analysis or comparison of the different approaches of the authors called Discussion is made, which then serve as a basis for making conclusions that punctuate the bibliographic findings, and to close the document, the bibliography which is the list of studies that have got the information.

Keywords: extensive grazing; production system; environmental effects; deforestation; physicochemical properties

\section{Introducción}

El panorama de la actividad ganadera, a través de los tiempos, ha generado una serie de efectos ambientales nocivos para el conjunto de los seres de la naturaleza, y por consiguiente ha degradado el suelo, es así que según estudios de Gerber et al (2013) agrega que esta situación, se plantea como uno de los dos o tres sectores con repercusiones más graves en los principales problemas medioambientales a todos los niveles, desde el ámbito local hasta el mundial. En Colombia la llegada de los colonizadores trajo consigo el cambio cultural en el sector agropecuario, lo que inicialmente condujo a la tala de bosques y posteriormente el cambio de pasturas, con las consecuencias que son el motivo de la realización de este artículo, en donde además se expondrán alternativas para prevenir o mitigar el impacto ambiental y social.

En consecuencia, la estructura del artículo, está constituida por un resumen que abarca en pocas palabras lo que trata el documento incluyendo las cinco palabras claves que se destacan en ese pequeño apartado; la introducción determinando la relevancia del tema en cuestión con algunos aspectos como el objetivo del mismo la metodología utilizada y demás; por otro lado, los resultados que consta de los registros documentales dados por autores expertos en la materia de la agroecología, igualmente interpretados en la discusión donde se confrontan los planteamientos de cada autor; las conclusiones que precisan el hallazgo documentan y por último la bibliografía de donde se extrajo la información.

Ahora bien, el objetivo principal de este artículo, es describir de qué manera la ganadería en Colombia es una actividad que genera impacto ambiental especialmente sobre el suelo, detallando a la vez, sus implicaciones. Así mismo, la ruta metodológica pertenece a la revisión documental desde el manejo de una base de datos extraída de documentos científicos especialmente de artículos, proyectos, y demás por vía internet; de igual manera, el tipo de investigación es la descriptiva porque se detallan los pormenores del impacto ambiental frente a la actividad ganadera en Colombia como también, es de enfoque analítico debido a que se analizan los hallazgos documentales.

\section{Metodología}

El tipo de investigación es la descriptiva, dado que, a través de la metodología de la revisión de documentos publicados en la red, en la internet, por su fácil acceso y gran cantidad de información, que demostraron el fenómeno planteado, y finalmente a través de un proceso de análisis de los resultados aportados por los investigadores, se confrontan sus hallazgos y conclusiones, lo que finalmente derivó en unas deducciones, que dieron el cierre al artículo.

Ahora bien, los criterios de búsqueda atendieron al estilo hermenéutico, en el cual se explica la universalidad de registros documentales sobre el impacto ambiental sobre el suelo debido a la actividad ganadera, pues se frecuenta una problemática grave en el contexto global.

Frente a las etapas o fases del estado del arte se trabajaron las siguientes: 
1-Etapa de Revisión Documental: Dada bajo fuentes de información desde el entorno científico-educativo de la ingeniería agroecológica, ya que es un encargo de los profesionales y demás cuerpo educativo fortalecer el sistema ambiental desde la actividad ganadera.

2-Etapa de selección y manejo de la información: donde se obtuvo la información para luego ser seleccionada tal como lo determinaba las categorías de estudio relacionándolas desde diferentes contextos, ya fuera el internacional, nacional y regional, puesto que todas ellas conservaron un mismo objetivo central el de ser $\mathrm{u}$ instrumento de estudio frente al impacto ambiental dejado por la ganadería.

3-Etapa de análisis de la información: en el que cada una de las investigaciones fueron analizadas y confrontadas unas con otras para extraer los aspectos concluyentes, cerrando así el estudio documental.

Desde otra esfera, las condiciones de impacto ambiental más relevantes derivadas de la actividad ganadera, redundan sobre el planteamiento de Sadeghian (2009) "la ganadería extensiva proporciona cambios negativos debido a la aridez del suelo, ya sea como producto de la deforestación en la creación de pastizales, al igual que por el pisoteo del ganado, que producen cambios estructurales, provocando detonantes erosivos, escases de nutrientes, entre otros, hasta la pérdida de diversidad de los mismos.

También esta actividad ocasiona efectos negativos del recurso hídrico, es decir, del agua. Tal como lo enuncia la Organización de las Naciones Unidas para la Agricultura y la Alimentación FAO (2006) "debido a los desechos animales entre ellos (estiércol y orines), se pierde la calidad del agua, conllevando a su polución, eutrofización y degeneración como se puede apreciar en los arrecifes de coral".

Al haber deterioro en el suelo, por consiguiente, afecta al medio ambiente, el cual ya no tendrá una densidad de oxigeno que augure la salud humana y animal, al igual que con la afectación de agua, se obtienen efectos adversos para las condiciones de vida de todos los seres vivos del planeta tierra.

\section{Resultados y discusión}

La tendencia actual a nivel mundial del uso de la tierra está enfocada en la conversión del entorno natural en pastos, en praderas de pastoreo, lo cual cambiará los datos de Fernández y Enríquez, donde dicen que la ganadería es la actividad que más utiliza los recursos de la tierra a nivel mundial. Ocupa aproximadamente el 30\% de la superficie terrestre libre de hielo. Aproximadamente el $80 \%$ de las tierras agrícolas están destinadas al pastoreo y producción de forrajes, lo que equivale a 3400 millones de hectáreas en el pastoreo y 500 millones en la producción de cultivos para alimentación del ganado.

En contraste con lo dicho, Las investigaciones actuales del Instituto Geográfico Agustín Codazzi ponen de presente que la ganadería extensiva se ha expandido sobre tierras apropiadas para usos agrícolas y forestales, trayendo como consecuencia la evidente subutilización e ineficiencia en el uso de los recursos, lo cual repercute en los campos social, económico y ambiental. (Ministerio de Ambiente, Vivienda y Desarrollo Territorial, 2007), y según Beltrán y Piñeros su avanzada la ha llevado incluso a áreas protegidas y parques naturales (2013).

\subsection{Marco conceptual}

Las características generales del ganado vacuno quedan descritas en su clasificación. Pertenece al orden Artiodactyla (mamíferos de número impar de dedos con cascos) y al suborden Ruminantia (estómagos divididos en cuatro compartimentos y con un número reducido de dientes, sin incisivos). Como otros miembros de la familia Bóvidos, tienen dos cuernos o astas huecos y sin ramificar que conservan durante toda la vida. Otros bóvidos están tan íntimamente emparentados con el verdadero ganado vacuno que aún pueden hibridarse entre sí, se divide en dos especies: Bovidae taurus, que tuvo su origen en Europa e incluye la mayoría de las variedades modernas de ganado lechero y de carne y Bovidae indicus, que tuvo su origen en India y se caracteriza por una joroba en la cruz (entre los hombros). Este último está muy extendido en África y Asia y en número menor, ha sido importado en América. 
La historia de Colombia y de Latinoamérica está ligada al tema de la ganadería, desde que Colón en su segundo viaje trajo 200 vacas flacas y maltratadas que encontraron unas condiciones propicias para sobrevivir, recuperarse y reproducirse. Beltrán (2003) luego se fueron dando las importaciones de diferentes razas.

La importación del ganado bovino a América desde su origen llegó con los conquistadores españoles como un elemento indispensable para su alimentación. Los pueblos indígenas no tenían animales domésticos, por consiguiente, sin el ganado hubiera sido imposible la conquista y apropiación del continente americano. Fedegan (2012) que como lo menciona esta federación, suena un tanto irónico, por lo de "apropiación".

Según lo relata Murgueitio (2003) la ganadería se inició en América aprovechando los ecosistemas de sabanas naturales presentes en varias regiones del Caribe, la Orinoquia y la Pampa argentina. Poco a poco avanzaría acompañando los desmontes de los bosques de los ecosistemas secos y húmedos, así como las laderas de las montañas y los altiplanos. Los animales ibéricos con el paso del tiempo se fueron adaptando a las nuevas condiciones y varios núcleos se transformaron en razas regionales llamadas criollas por los expertos.

Desde el ámbito de la ganadería en Colombia, se tiene noticia de las primeras reses introducidas por Rodrigo de Bastidas en 1525 con destino a su gobernación de Santa Marta. Desde la ciudad primada se esparcieron por el país con las expediciones de la conquista. Sourdis (2008) luego se dieron los cruces con sus nuevas razas y las que se determinaron como autóctonas de nuestro país.

Por otro lado, a lo que respecta a las áreas de ganadería extensiva en el país, se destaca el estudio ejecutado por el Instituto Amazónico de Investigaciones Científicas (SINCHI, 2006), al agregar que:"El territorio Amazónico representa el mayor porcentaje de bosques naturales para Colombia, mas sin embargo, las selvas amazónicas la están acabando para transformarla en pastizales hacia la búsqueda de la ganadería extensiva y semiextensiva. Igualmente, entre los departamentos más deforestados se halla el Caquetá al representar cifras que producen realmente espanto, donde de los 16 municipios que lo conforma, ocho (8) de ellos tiene más del 50\% de su superficie terrestre destinada a pastizales: Albania (98\%), Curillo (57\%), El Doncello (51\%), Paujil (58 \%), La Montañita (63\%), Milán (69\%), Morelia (98\%), Solita (89\%).

Frente a lo expuesto es importante aclarar que la ganadería en el país decae en la actualidad, ya que se ha perdido totalmente su figura destacable frente a las demás actividades económicas. Según el Ministerio de Agricultura y desarrollo rural (2009) "el área brindada a la ganadería, se convierte en una cifra, nueve veces mayor que el de la función agrícola; por lo tanto representa el $67 \%$ por encima del índice de producción pecuaria y el $30 \%$ de la obtención agropecuaria; así mismo, pertenece al doble del ejercicio avícola, como también, triplica la producción cafetera, en una quinta parte la producción de flores y seis veces la producción de arroz, lo que indica que la ganadería fue la fuente económica más significativa para el país".

Los indicadores de productividad ganadera, presenta deficiente desempeño tecnológico, así Vergara (2010) expresa "El área en ganadería es de 38 millones de hectáreas, a partir de una capacidad de carga de 0,6 cabezas/ha, esta cifra ubica a la ganadería como una producción extensiva" igualmente, el denominado hato ganadero colombiano, lo constituye una cifra aproximada de 25 millones de cabezas de ganado, la cual ha sido una constante durante 15 años. Por su parte, la producción cárnica es del 55\%, el $4 \%$ a lechería y el $40 \%$ a ganado doble propósito. En consecuencia, se tiene una mayor tendencia a la cría de hembras, donde en el hato $64 \%$ son hembras y el $35 \%$ machos".

De acuerdo a los estudios de la OCDE y la FAO (2012) "En la actualidad el entorno de productividad alimenticia, ha generado la necesidad en el mundo de producir más alimentos para una población creciente, los especialistas, los productores, se enfrentan al reto de la generación de soluciones para disminuir el impacto negativo de cualquier actividad agrícola o pecuaria, sobre la degradación del suelo, agua y aire, al mismo tiempo que se incrementa la presión sobre estos recursos naturales", por lo tanto, existe una marcada preocupación por la escasez de los recursos naturales, la cual, fue expuesta bajo una primicia sistemática por Malthus, quien analizando la rápida explosión demográfica y su relación con la producción de recursos alimenticios planteó que "mientras la población crece en proporción geométrica, la producción alimenticia lo hace en proporción aritmética". 


\subsection{Tipos de explotación Ganadera}

En Colombia, se pueden visualizar tres tipos de explotación ganadera, que son utilizados por los productores pecuarios y agropecuarios dependiendo de los recursos económicos y tecnológicos disponibles, los cuales son: Explotación extensiva y explotación intensiva y últimamente se ha implementado la explotación mixta (Figura. 1).

\subsubsection{Ganadería Extensiva}

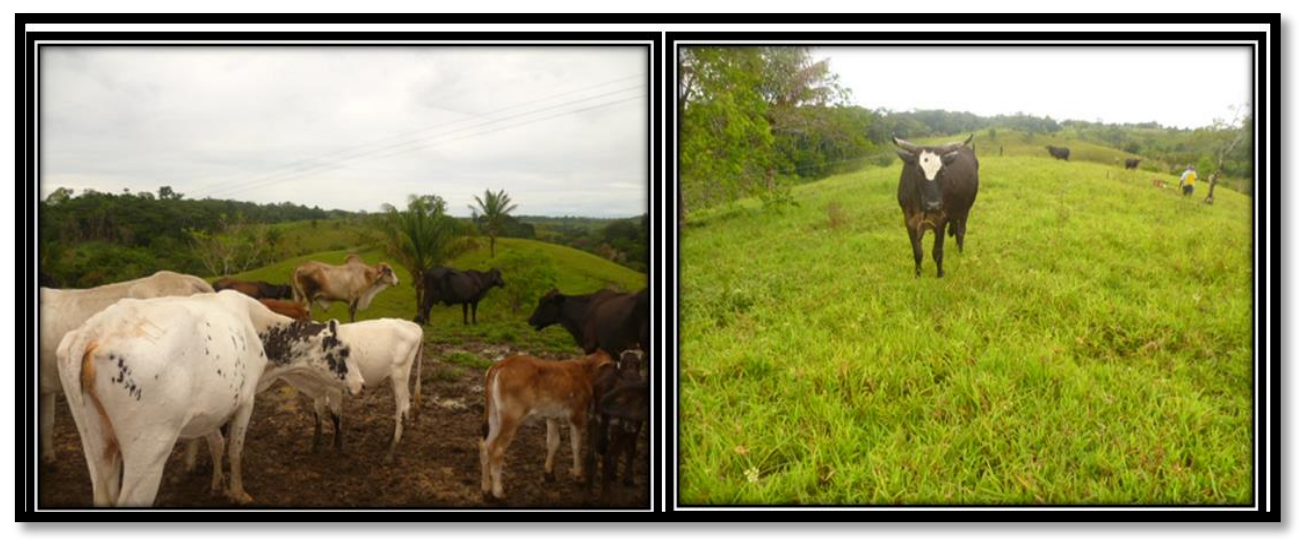

Figura. 1. Deforestación para la implementación de pasturas en zona rural de Valparaíso Caquetá año 2016

Es la ganadería tradicional en donde se encuentran grandes cantidades de terreno con pocos animales que se alimentan directamente de lo que produce el terreno (FINAGRO, 2009), de los recursos que se encuentran en él sin un mejoramiento de praderas (UNAD, sf). No hay cercado en todo el potrero., (Mahecha, Gallego, \& Pelaez, 2002) .Por otra parte, según la investigación realizada por el Instituto Amazónico de Investigaciones Científicas (SINCHI, 2006), se afirma, que a pesar de que la Región Amazónica representa el mayor porcentaje de bosques naturales para Colombia, estos están siendo transformados para pastizales con fines de ganadería extensiva y semiextensiva. Entre los departamentos más deforestados se encuentra el departamento del Caquetá con cifras alarmantes, en donde de los 16 municipios que lo conforma, ocho (8) de ellos tiene más del 50\% de su superficie terrestre destinada a pastizales: Albania (98\%), Curillo (57\%), El Doncello (51\%), Paujil (58 \%), La Montañita (63\%), Milán (69\%), Morelia (98\%), Solita (89\%). Estos porcentajes en parte se deben a que por historia y tradición, la ganadería es la principal fuente de ingresos para el sostenimiento de muchos Caqueteños, y además es la economía que mueve el departamento, afirmación que se refleja en las estadísticas del Comité de Ganaderos del Caquetá S.A., que indica que en el primer trimestre de 2012 se ascendió a 27.843 cabezas comercializadas en el departamento y hacia el interior del país, con un aumento anual de 2.163 ejemplares correspondiente al 8,4\% (Líder, 2012).

\subsubsection{Ganadería Intensiva}

Este tipo de ganadería es la que se desarrolla con fines productivos altos, utilizando las últimas tecnología o tecnología de punta; para llevar al mercado nacional e internacional, carne, leche, pieles, lana, huevos entre otros. La característica fundamental es que los animales son estabulados y confinados en espacios que generalmente son adecuados bajo condiciones de temperatura, luz y humedad que han sido creadas en forma artificial, con el objeto de incrementar la producción en el menor tiempo posible, minimización de espacio, de fuerza de trabajo y optimizando el control de manejo, nutrición y producción. (Mahecha, Gallego, \& Pelaez, 2002)

Este tipo de ganadería es la que se desarrolla con fines productivos altos, utilizando las últimas tecnología o tecnología de punta; para llevar al mercado nacional e internacional, carne, leche, pieles, lana, huevos entre otros. La característica fundamental es que los animales son estabulados y confinados en espacios que generalmente son 
adecuados bajo condiciones de temperatura, luz y humedad que han sido creadas en forma artificial, con el objeto de incrementar la producción en el menor tiempo posible, minimización de espacio, de fuerza de trabajo y optimizando el control de manejo, nutrición y producción. OCDE y la FAO (2012).

\subsubsection{Sistemas de explotación mixta}

Es aquella en la que se tienen los animales un tiempo en estabulación y otro en potrero. Esto se hace con el fin de manejar la alimentación en las etapas de engorde y de lactancia. También es importante en el manejo de renovación de praderas y el de darle un bienestar a los animales al sentirse libres, se estresan menos y producen más. (OCDE y la FAO (2012).

El tipo de ganadería predominante en Colombia es la extensiva, los ganaderos han convertido zonas que se suponían inaccesibles para el hombre, en praderas para el ganado, donde además de la acción del hombre sobre el ecosistema, se han dado las de los mismos animales, no solo sobre el suelo, sino en el aire y el agua.

\subsection{Efecto de la ganadería sobre las características del suelo}

De acuerdo a los estudios de FAO (2002) "Se ha detonado una mayor producción y exportaciones de granos en Países como el Brasil y Colombia, donde las tierras abundan relativamente, en parte a consecuencia de la deforestación por el proceso de la actividad ganadera." Esto indica, la gravedad del impacto ambiental generado por la acción ganadera donde se ha reducido la producción de alimentos (Figura. 2).

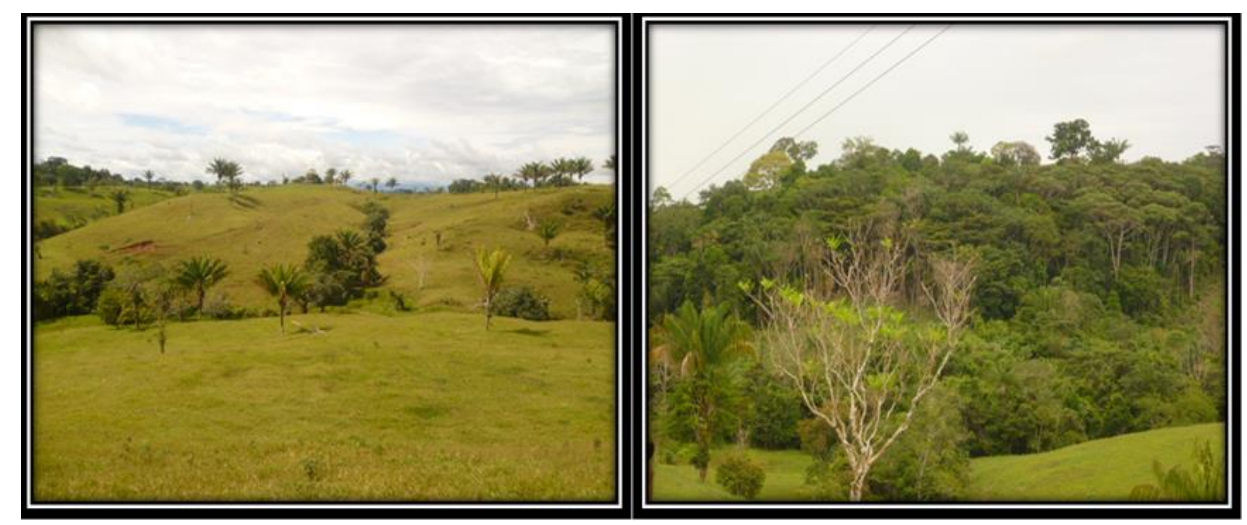

Figura. 2. Impacto ambiental del proceso de deforestación para la implementación de pasturas en zona rural de Valparaíso Caquetá año 2016

Igualmente, Gaitán, Juan José et al 2009: en su estudio sobre la evaluación de la composición florística y estructura espacial de la vegetación, del estado de la superficie del suelo en los interparches, se halló un sistema de degradación difícil de superar, y que a la vez compromete a los demás ecosistemas.

También podemos mencionar que debido al constante paso de grandes cantidades de animales resulta una masa compactada que como resultado genera deslizamientos, avalanchas y derrumbes, a esto le sumamos el transito usado para el transporte de leche aumentando los daños, por estas dos razones nos encontramos con un suelo débil y poco fértil, causando de esta manera que la labor de la ganadería sobrepase los costos normales (Rivera 2001).

A su vez, es importante llevar a colación lo aportes de Cuesta et al 2005 y Chaparro 2005 quienes aseguraron que en los "suelos tropicales en ganadería el pisoteo produce una presión aproximada de $9 \mathrm{kgcm}^{-2}$ (según origen del suelo y la localización en el perfil) a su vez, Cuesta, et al., 2005); el parámetro más utilizado para cuantificarla es la densidad aparente, es de utilidad en términos porcentuales a partir de mediciones estandarizadas igualmente Chaparro (2005), está relacionada con el contenido de humedad y correlaciona inversamente con porosidad y propiedades hidráulicas 
del suelo. Esta evaluación concreto el problema de como el pisoteo del ganado afecta las condiciones de vida de los demás animales.

En este sentido, se halla la posibilidad de que la ganadería puede jugar un papel importante en el mantenimiento de la fertilidad del suelo, sobre terrenos pequeños o cerrados, más en grande extensión lo que se tiene es un efecto perjudicial tanto para el suelo como para los mismos animales del hato. De lo mencionado, los efectos adversos sobre el suelo los dividiremos de acuerdo a su origen directo (del animal por pisoteo y excreciones) e indirecto (del hombre sobre el terreno para el establecimiento de la ganadería, así como de lo que le aporta al animal).

Entre los efectos Directos se halla la repercusión en las propiedades químicas y físicas del suelo. Otros son los cambios en cuanto a sus propiedades químicas (disponibilidad de macro y micronutrientes), como son micro y macroelementos, el Nitrógeno, el Fósforo, Potasio, como la liberación de carbono de los depósitos de materia orgánica, mencionados por Basso et al (2009); Cuenca (2010) así como la salinización del perfil, hallazgo de (Andriulo et al., 2003).

Las afectaciones sobre las propiedades físicas, la degradación de la vegetación, la erosión del suelo, mencionada por Baridón y Cattani y Fernández y Enríquez, la desertización y compactación del mismo FAO (2006), efectos producidos sobre la pastura (defoliación, tránsito y pisoteo, retorno por excretas) y sobre el suelo (tránsito y pisoteo) Coronel (2015), los procesos erosivos, cambios en el horizonte 0 y la estructura, lo que conlleva a afectar la fertilidad química, mencionado por (Vargas, et al., 2003).

Ambos interactúan con los microorganismos del suelo, los cuales intervienen en el reciclado de nutrientes aportados por los residuos vegetales y animales del sistema pastoreado., Díaz, et al., (2008), Hofstede (2004), Sepúlveda (2008) y Taboada (2007) así como en su estructura dimensional, según los estudios de (Pérez, et al., 2009).

Inicialmente, para el establecimiento de un sistema de ganadería extensivo, el hombre debe cambiar la cobertura vegetal, por lo tanto, su primera intervención es la de talar o cortar el bosque nativo, para luego mediante diversos procesos químicos o mecánicos (quema), sembrar nuevas especies de pastos. Este efecto del desmonte genera el primer impacto ambiental (Ambrogi, 2000); (Gutiérrez, 2007); (Núñez y Núñez 2014).

Desde otra esfera, las condiciones de impacto ambiental más relevantes derivadas de la actividad ganadera, redundan sobre el planteamiento de Sadeghian (2009):

"La ganadería extensiva proporciona cambios negativos debido a la aridez del suelo, ya sea como producto de la deforestación en la creación de pastizales, al igual que por el pisoteo del ganado, que producen cambios estructurales, provocando detonantes erosivos, escases de nutrientes, entre otros, hasta la pérdida de diversidad de los mismos".

Los efectos sobre el agua se relatan en otros informes como los de Alfaro y Salazar, que dejan claro que la contaminación de este vital elemento se hace directamente por las sustancias agrotóxicas empleadas en el proceso de producción ganadera, así como a través de las secreciones de estos animales. El efecto adverso del bovino directo sobre el suelo se ve potencializado por la excreción de residuos de medicamentos como purgantes, antibióticos, entre otros, pues afectan las especies animales del suelo, encontrado por Martínez y Cruz 2000, que según Espejo y García 2001 se pueden disminuir con dieta especial.

Es evidente que las afectaciones sobre el agua, el suelo y el aire, tienen su consecuencia también sobre la biodiversidad, pues se altera su ecosistema y sus fuentes de alimentación (Acosta y Guevara, 2009); (Castro, 2007); (Eastman y García, 2007)

La práctica de quemas estacionales promueve la uniformidad genética al privilegiarse el monocultivo de gramíneas y eliminación de la sucesión vegetal por medios químicos (herbicidas) o físicos (Bernardis, et al., 2004); la desecación de humedales; la construcción de vías de penetración (Cotto, 2012).

Frente a la discusión de los autores citados que hablan del tema en cuestión y ante la problemática observada, está claro, según Vera 2008, la situación actual del sector ganadero demanda la aplicación de modelos alternativos que 
reúnan componentes agroforestales y la actividad pecuaria con el fin de mejorar la producción y a la vez disminuir los impactos al ecosistema, generando beneficios ambientales.

Dependiendo de cuál sea el ecosistema afectado (suelo, agua o aire), las soluciones que se arbitren deberán hacerse de forma integrada. Hay que saber qué residuos se producen y sobre los que se puede incidir, ya sea para no generarlos, ya sea para reducirlos o para rentabilizarlos. Inicialmente, el Ministerio de Agricultura y Desarrollo 2015, a través del Decreto ley 2364 de 2015, mediante el cual crea la Agencia de Desarrollo Rural con el objeto de ejecutar la política de desarrollo agropecuario y rural con enfoque territorial formulada por este ministerio, trata de regular la tenencia de tierra y su disposición, con énfasis en la sustentabilidad y sostenibilidad. Igualmente. Vera (2008) con la zoo-cría de avestruces, que es un ejemplo nada más de varias elecciones.

Cabe resaltar que la ganadería bovina ocupa la mayor parte de las tierras explotadas de Colombia y desarrolla actividades, como la tala y la quema de bosques, la uniformidad genética al privilegiarse el monocultivo de gramínea, la desecación de humedales, la construcción de vías de penetración, la demanda creciente del daño directo madera para construcciones, que generan impactos de especial consideración sobre los recursos naturales y el medio ambiente, lo que puede conllevar a desequilibrios naturales de considerable importancia. (Mahecha L.L2000).

Así mismo, los procedimientos de renovación hacia praderas y sistemas silvopastoriles implica mejorar las condiciones alimentarias de los animales, así como en la conservación de los suelos, procesos de reciclaje de nutrientes, circulación y aprovechamiento de agua en el sistema y biodiversidad, según Piza et al (2001) el silvopastoreo Chamorro (2004); Mahecha (2002); Mahecha (2000); Solorio et al (2009) establecen un sistema de producción pecuaria en donde las leñosas perennes (árboles y/o arbustos) interactúan con los componentes tradicionales (forrajeras herbáceas y animales) bajo un sistema de manejo integral, ha generado unos resultados científicos como alternativa de producción sostenible que promueve a la reducción del impacto ambiental de los sistemas tradicionales de producción, resulta una manera de tratar la situación problemática.

Existen varios estudios para solucionar el problema de deterioro medioambiental, está la de Arelovich(2012), quien plantea adoptar una tecnología especializada para producir en zonas semiáridas, o la nanotecnología en suelos contaminados, según Carrillo y González (2008)., la fertilización de pasturas Marino y Agnusdey (2007) y una aún más radical, es la de un modelo de producción animal alternativo, como el de Vera (2008) con la zoocría de avestruces, ello dado como un ejemplo a seleccionar para erradicar el impacto ambiental que proporciona la ganadería extensiva.

Adentrándonos, en el deterioro del suelo, se ha presentado la propuesta alternativa sobre la aplicación de fertilizantes minerales en los cultivos, reemplazando la técnica de arrojar abonos orgánicos solos. Sin embargo, al combinar estos dos tipos de fertilizantes se liquidan las desventajas específicas de las dos clases de abonos, creándose así las condiciones de su aprovechamiento más racional (Peña, 2000); (Cordero, 2010).

Es importante resaltar que dentro de las posibles soluciones a la problemática medioambiental en cuestión, se puede adoptar la medida recomendada por Matthews 2006, integrante de la Oficina de prensa de la FAO (2008) quien persigue que se debe hacer una degradación del suelo, bajo controles permanentes que vigilen los accesos y la eliminación de los impedimentos que conduzcan a una efectiva movilidad en los pastos comunales", lo que indica que es factible la utilización de medidas o métodos que promuevan a la conservación del suelo y el silvopastoreo, así como también, a la exclusión controlada del ganado sobre terrenos o áreas delicadas y las acciones que conlleven al pago por servicios medioambientales en el uso del suelo en la ganadería para limitar su degradación. Por lo tanto, este proceso de resarcir el deterioro del suelo por efectos de la actividad ganadera es valiosa y comprende un alcance que amplía las posibilidades de reducir la situación medioambiental, en procura de mejorar este desempeño agropecuario.

Ante lo dicho, existe otro tanto de autores que piensan que la implementación del silvopastoreo, es un sistema de producción pecuaria en donde las leñosas perennes (árboles y/o arbustos) interactúan con los componentes tradicionales (forrajeras herbáceas y animales) (Solorio, Bacab, Castillo, Ramirez, \& Casanova, 2009) bajo un sistema de manejo integral, (Mahecha, 2002) ha sido planteado con base en resultados investigativos, como una 
alternativa de producción sostenible que permite reducir el impacto ambiental de los sistemas tradicionales de producción, nos demuestran que es una muy buena alternativa para contrarrestar el impacto ambiental generado por la ganadería. .

Actualmente el Modelo de Evaluación Ambiental de la Ganadería Mundial (GLEAM) es un entorno de modelización que simula la interacción de los procesos y actividades de producción ganadera con el medio ambiente. GLEAM se ha desarrollado para evaluar tanto el impacto de la ganadería como diferentes opciones de adaptación y mitigación a escala (sub)nacional, regional y global. (FAO, sf)

En este sentido, Fernández y Mayoralas 2009, agregan que el gobierno central no ha brindado los recursos económicos para mitigar el impacto ambiental producido por la ganadera.

\section{Conclusiones}

La problemática ambiental generada por la ganadería extensiva en Colombia es bastante preocupante, por cuanto los suelos se vienen afectando aceleradamente, desde todos sus componentes fisicoquímicos, lo que indica, que más que un estudio a las condiciones de impacto ambiental, este artículo representa un S.O.S. convocando a un llamamiento urgente de atender, en donde los entes gubernamentales en sus políticas de estado deberían implementar de medidas que conlleven, a minimizar la problemática, o a evitar su proliferación, ante el riesgo de perder un gran porcentaje de productividad alimentaria.

Los estudios recientes ofrecen alternativas de producción animal, que no presentan las desventajas de la ganadería, sobre su impacto ambiental, y que no solamente favorecen este aspecto, sino que conllevan a menores costos de inversión y producción. Mientras el gobierno no legisle en cuanto a estándares de daño o contaminación mínimos permitidos por las empresas ganaderas, se seguirá expandiendo esta frontera, como lo ha venido haciendo, hasta zonas de protección, parques naturales.

La ganadería en Colombia y en el mundo, ha cambiado el sistema de producción agrícola tradicional, por el de pastoreo, para lo cual se dan cambios graves en el suelo, el paisaje, el aire, los ríos y la biodiversidad, también presentando consecuencias sociales y políticas.

Los productores del sector pecuario deben implementar alternativas amigables con el medio ambiente, así como emprender campañas de recuperación de ecosistemas.

La implementación de sistemas silvopastoriles, otros tipos de producción, al final será la única forma de evitar un mayor daño en los ecosistemas.

El gobierno deberá, a través de leyes, ser más estricto y ejercer mayor control sobre los productores ganaderos, así como a quienes implementen prácticas agrícolas que conlleven al deterioro del medio ambiente sobre el suelo, aire agua y la biodiversidad, además de la afectación en la biodiversidad y la salud humana.

\section{Referencias bibliográficas}

Ambrogi, R. (2000). La actividad ganadera y su impacto en la deforestación: estudio de caso en la zona de Bocao, Chontales y Nueva Guinea (Nicaragua). Obtenido de http://www.sidalc.net/cgi-

bin/wxis.exe/?IsisScript=rednia.xis\&method=post \&formato $=2 \&$ cantidad $=1 \&$ expresion $=\mathrm{mfn}=014$

Arelovich, H. M. (2012). Tecnología disponible de potencial impacto en la ganadería. Obtenido de http://sedici.unlp.edu.ar/bitstream/handle/10915/27603/Documento_completo.pdf?s

Arias Jimenez, A. C. (2001). Suelos Tropicales. Costa Rica: Universidad Estatal a Distancia.

Beltrán F., J., \& Piñeros, M. A. (2013). Sector agropecuario colombiano: su realidad económica y perspectiva. Consultado en. http://repository.ean.edu.co/bitstream/handle/10882/4629/BeltranJorge2013.pdf?sequence=1 
Carrillo G., R., \& González Ch., M. (2008). La nanotecnología en la agricultura y rehabilitación de suelos contaminados. Obtenido de http://carmenglez.galeon.com/principal/PDF

Chaparro, O. 2005. Medición de las propiedades mecánicas de un suelo agrícola. Documento preliminar elaborado como material de apoyo para la asignatura de Física de suelo. Palmira: Universidad Nacional de Colombia. Recuperado el 27 de mayo de 2017 de http://www.scielo.org.co/scielo.php?script=sci_arttext\&pid=S012028122009000200004

CORPOAMAZONIA.2001 (s.f.). Region del Caqueta. Recuperado el 13 de Marzo de 2017, de Sistema Económico: http://www.corpoamazonia.gov.co/region/Caqueta/Caq_Economico.htm\#

Cuesta et al 2005 Procesos tecnológicos para la renovación de praderas degradadas en las regiones caribe y valles interandinos. Corpoica. Recuperado el 27 de mayo de 2017 de www.corpoica.org.co

(DANE), D. A. (2005). Municipio de Milán. Recuperado el 02 de Marzo de 2017, de Municipio de Milán: http://www.corpoamazonia.gov.co/region/Caqueta/Municipios/Caq_Milan.html

FAO (2002). Ganadería y deforestación, Recuperado el 27 de mayo de 20|17 de www.fao.org/3/a-a0262s.pdf

FAO. (2006). La ganadería amenaza el medio ambiente. Obtenido de http://www.fao.org/Newsroom/es/news/2006/1000448/index.html

FAO. (2012). Medir mejor el impacto ambiental de la ganadería. Obtenido de http://www.fao.org/news/story/es/item/150751/icode/

FAO 2017. (sf) . Modelo de Evaluación Ambiental de la Ganadería Mundial (GLEAM). Obtenido de http://www.fao.org/gleam/es/

FAO., 2008. Ganadería: una amenaza para el medio ambiente. Ecosofia.org. Consultado el 12 de Marzo de 2017. http://ecosofia.org/2008/02/ganaderia_amenaza_medio_ambiente.html

FAO., 2006. La larga sombra del ganado. Consultado en. http://www.fao.org/docrep/011/a0701s/a0701s00.htm

Fedegan., 2012. Historia de Fedegan. Consultado el 15 de Marzo de 2017. http://www.fedegan.org.co/quienessomos/nuestra-historia

Fernández, E., \& M., E. (2010). Metodologías para la evaluación y mejora de los sistemas ganaderos: análisis comparado y posibilidades de aplicación en el sector de los pequeños rumiantes de Andalucía. Obtenido de http://www.uco.es/zootecniaygestion/img/pictorex/20_10_02_Trabajo_Fin_de_Master.pdf

Gaitán, Juan José et al 2009: Efectos del pastoreo sobre el suelo y la vegetación en la Estepa Patagónica. Recuperado el 27 de mayo de 2017 de http://www.scielo.org.ar/scielo.php?script=sci_arttext\&pid=S185020672009000200013

Gerber, P. W. et al,.(2013). Enfrentando el cambio climático a través de la ganadería - Una evaluación global de las emisiones y oportunidades de mitigación. Organización de las naciones unidas para la alimentación y la agricultura (FAO), Roma. 
Gutierrez, C, 2007. Análisis del impacto ambiental de las actividades agropecuarias sobre el suelo, agua y bosque, en la comunidad de "El Poxté", Poplún, Petén, Consultado en.

http://www.sidalc.net/cgibin/wxis.exe/?IsisScript=GREYLIT.xis\&method=post\&formato=2\&cantidad=1\&expres.

Mahecha, L. (2000). El silvopastoreo: una alternativa para la producción bovina sostenible y competitiva Obtenido de: http://rccp.udea.edu.co/index.php/ojs/article/view/90

Mahecha, G. L. A. et al, (2002). Situación actual de la ganadería de carne en Colombia y alternativas para impulsar su competitividad y sostenibilidad. Obtenido de

http://datateca.unad.edu.co/contenidos/116001/Material_Unidad_II/GANADERIA_DE_CARNE_EN_COLOMBI A.pdf

Minagricultura. (2012). Recomendaciones ganadería de carne y leche. Obtenido de

http://portalmadr.minagricultura.gov.co/Cambio_Climatico/Documents/recomendaciones\%20ganaderia\%20de\%20 carne $\% 20 \mathrm{y} \% 20$ leche.pdf\#search $=\mathrm{g}$ anader\%C3\%ADa

Ministerio de Agricultura y Desarrollo Social. (2015). Decreto ley 2364 de 2015. Obtenido de http://es.presidencia.gov.co/normativa/normativa/DECRETO\%20182\%20DEL\%2005\%20DE\%20FEBRERO\%20 DE\%202016.pdf

Ministerio de Ambiente, Vivienda y Desarrollo Territorial. (2007). Panorama sobre el uso y ocupación actual de las tierras en Colombia. Obtenido de cedins.org/.../29-panorama-sobre-el-uso-y-ocupacion-actual-de-las-tierra.

Moreno, C.E., 2001. Métodos para medir la biodiversidad. M\&T-Manuales y Tesis SEA, vol.1.Zaragoza, 84pp.

Murgueitio, E., 2003. Impacto ambiental de la ganadería de leche en Colombia y alternativas de solución. Fundación Centro para la Investigación en Sistemas Sostenibles de Producción Agropecuaria (CIPAV).

Nuñez, Z. R., Nuñez Z., M., 2014. Explotacion ganadera-cría y engorde de ganado vacuno. Consultado en: http://www.seam.gov.py/sites/default/files/users/control/marcela\%26ruben nu\%C3\%B1ez_adolfo.a.pdf

OCDE y la FAO (2012). Nuevo informe sobre las perspectivas de la agricultura mundial. Recuperado el 27 de mayo de 2017 de http://www.fao.org/news/story/es/item/151319/icode/

Piza, P. (2001). Estado del arte de algunos sistemas de producción ganadera de clima frío en Colombia y el mundo. Obtenido de: http://biblioteca.uniminuto.edu/ojs/index.php/Inventum/article/view/384

Sadeghian, Kh. S.,(2009). Impacto de la ganadería sobre el suelo. Obtenido de alternativas sostenible de manejo: http://www.establo.info/impacto\%20de\%20la\%20ganaderia\%20sobre\%20el\%20suelo.pdf

SINCHI, I. A. (2006). Balance anual sobre el estado de los ecosistemas y el ambiente de la amazonia colombiana. SINCHI. Bogotá, D.C: Instituto Amazónico de Investigaciones Científicas -Sinchi.

Sourdis, A., 2008. Ganadería en Colombia: cinco siglos construyendo país. Consultado el 15 de Marzo de 2017. https://books.google.com.co/books/about/Ganader\%C3\%ADa_en_Colombia.html?id=0I1CQwAACAAJ 
https://doi.org/10.25054/issn.2216-1325

Vergara Vergara, W. (2010). La ganadería extensiva y el problema agrario. Obtenido de El reto de un modelo de desarrollo rural sustentable para Colombia: http://revistas.lasalle.edu.co/index.php/ca/article/viewFile/350/281 